\title{
CRC Screening: Is It Worthwhile in Younger Adults?
}

\author{
Ethan Bortniker $^{3} \cdot$ Joseph C. Anderson ${ }^{1,2,3}$
}

Published online: 2 January 2016

(C) Springer Science+Business Media New York (Outside the USA) 2015

\begin{abstract}
Although current guidelines recommend screening for colorectal cancer (CRC) in average-risk individual starting at age 50 [1], more than $10 \%$ of CRC is diagnosed prior to this age [2]. Furthermore, while the rates for older individuals have decreased, the rates for younger adults may be increasing [2]. Current guidelines recommend that individuals with a family history of CRC or a genetic syndrome should be screened at an age younger than 50 years, preferentially with colonoscopy [1]. In 2009, the American College of Gastroenterology (ACG) recommended colonoscopic screening of African-Americans starting at age 45 [1]. Can other groups of adults who may be at risk of CRC at an early age be identified? In the current issue of Digestive Diseases and Sciences, Jung et al. [3] describe a strategy designed to identify high-risk individuals $<50$ years old who might benefit from screening colonoscopy and thus reduce the substantial burden of colorectal cancer in this age group.

Jung et al. report a cross-sectional analysis of 27,894 Korean adults who underwent an initial colonoscopy for preventative health care. The investigators collected data that included age, sex, height, weight, medications, family
\end{abstract}

The contents of this work do not represent the views of the Department of Veterans Affairs or the United States Government

Joseph C. Anderson

joseph.anderson@dartmouth.edu

1 Department of Veterans Affairs Medical Center, White River Junction, VT, USA

2 The Geisel School of Medicine at Dartmouth, Hanover, NH, USA

3 Division of Gastroenterology and Hepatology, University of Connecticut School of Medicine, Farmington, CT 06030, USA history of colorectal neoplasia, smoking exposure, alcohol intake, physical activity, self-reported comprehensive medical and surgical history, and blood pressure measurements. Laboratory data collected included hepatic sonographic data, serum concentrations of triglyceride, cholesterol, and glucose in addition to screening colonoscopy reports. Using these data, the authors stratified the participants by risk factors including sex and age. The investigators then calculated the number needed to screen (NNS) to identify one adult with advanced colorectal neoplasm for each group. The NNS was calculated as the inverse of the prevalence of advanced lesions for each group. Thus, a group with a lower NNS might derive greater benefit from screening colonoscopy than a group with a higher NNS. They compared the NNS in men, stratified into age groups under 50 years as well as varying risk exposures, to those of a low-risk group of women 50 years and older with no known risk factors.

The investigators observed that the only group with a lower NNS than the control group of women over age of 50 years $(26.4$; $95 \%$ CI 19.0-43.5) was the cohort of men 45-49 years old who had all of the risk factors considered by the authors: smoking, metabolic syndrome, obesity, and fatty liver disease (18.4; $95 \%$ CI 12.6-34.1, range 16.9-22.9). Thus, they concluded that there was no benefit to screening high-risk individuals $<45$ years old. Do these data suggest that the effort to find a high-risk group in young adults may be futile?

There are many strong points in the current study, which help to support its findings. The major strength is the sample size, which included over 25,000 individuals younger than 50 years, a group that has not been included in most of the previously published screening studies. Furthermore, the exclusion of adults with a family history of CRC is also important since these individuals are 
already recommended to have screening prior to 50 years of age. Furthermore, the investigators screened the participants with a thorough history and physical examination to ensure that they were asymptomatic prior to colonoscopy. The authors also include two important CRC risk factors, obesity and smoking, as part of their strategy, factors which have been highlighted by the 2009 ACG CRC screening guidelines as potentially important for the identification high-risk individuals [1]. Further, obesity [4] and smoking [5] have also been identified as important factors in large cross-sectional studies and advanced adenoma prediction models developed in older populations [6, 7]. Finally, the authors combined lifestyle factors with biologic-based factors in the form of commonly ordered laboratory tests.

Further insight into the current study can be gained from examining a previously published analysis of the same cohort. There were only 1,155 subjects with a family history of CRC excluded from the current sample [8]. One interesting observation is that the same investigators arrive at somewhat different conclusions based on similar findings. In the first analysis, the authors observed that there was no statistical difference in the prevalence of advanced adenomas between the men aged 30-39 with all of the risk factors $(1.7 \%$; calculated $\mathrm{NNS}=58.8)$ as compared to women older than 50 years $(3.8 \%$; calculated NNS = 26.3) $(p=0.184)$. The authors conclude that their results suggest that persons with certain risk factors might benefit from screening colonoscopy before the age of 50 years. In the current publication, the authors conclude that since the NNS for an advanced adenoma $(55.4$; prevalence $=1.8 \%$ ) in men aged 30-39 is higher than that for women 50 years or older $(26.4$; prevalence $=3.8 \%$ ), there is no benefit to screen adults with these risk factors prior to age 45 . Aside from the statistical consideration, the fundamental message is that the interpretation of the results of these studies can be subjective. One possible solution, as suggested by the authors, is to use a benchmark. The authors of the current study as well as other investigators [9] have suggested an acceptable NNS cutoff of 25, based on a study by Imperiale et al. [10]. Using this benchmark, the data of the current study suggest that screening in men aged 30-44 years, even those with all commonly accepted risk factors, is not warranted.

Although the same extensive questionnaire was presumably used in both studies, the current study reports on fewer risk factors such as exercise or alcohol use. This exclusion in the current paper may perhaps be due to the lack of association between these other factors and colorectal neoplasia across all of the age groups, as published previously [8]. One potentially important difference with respect to risk factors is how the two studies examined smoking as a variable. Whereas the previous analysis used a cutoff exposure of 20 pack-years, the current analysis used a lower exposure of 10 pack-years. Of note, in the previous study, adults in the 30-39-year age group who smoked 20 or more pack-years had over a fourfold risk (adjusted odds ratio 4.41; $95 \%$ CI 1.80-10.80; $p<0.001$ ) for advanced neoplasia as compared to those with a lower exposure. Smoking, at even higher exposure levels of 30 or more pack-years, has a twofold risk of adenomas in young and older adults $[5,7,11]$. Other similar studies have also documented that smoking is an important predictor in younger adults when using a definition of current smoking as an exposure variable $[12,13]$. Thus, it is possible that the stratification of smoking exposure may have had an impact on the outcome.

But it is also possible that like the prediction models, these lifestyle factors may not be potent predictors. The prevalence of advanced adenomas for males across the age groups $30-39,40-44,45-49$, and $\geq 50$ years old was 0.7 , $1.9,4.1$, and $9.1 \%$, respectively ( $p$ for trend $<0.001$ ). The substantial increase in risk, especially at $\sim 50$ years of age, supports the observation that age is the strongest predictor of colorectal neoplasia [14]. The addition of lifestyle risk factors, including strong risk factors such as smoking or obesity, has added little to advanced adenoma prediction models in older individuals [14]. This may limit the possibility of identifying a high-risk group in young adults using these lifestyle factors alone.

There are some other important factors, which may limit the generalizability of this study's findings. The study sample consisted of an ethnically homogenous population, Korean adults who had undergone screening colonoscopies. Moreover, since a fraction of subjects, due to free availability, chose to undergo colonoscopy regardless of current guidelines, many adults significantly younger than 50 years were included in the analysis. Nevertheless, many of these younger subjects might be considered self-selected, further limiting generalizability. The current study also used a BMI of 25 as a cutoff for obesity as opposed to a BMI of 30, the latter of which is used in studies performed in Western populations [4]. Thus, these data may not be applicable to the heavier population of the USA. Another issue of generalizability is related to the overall effectiveness of colonoscopy as measured by quality indicators such as withdrawal time, colon preparation quality, and the endoscopists' adenoma detection rates. Though this study does not report an adenoma detection rate, the previous study by the same authors reported a relatively robust $30.1 \%$ for all subjects 50-59 years of age [8]. The previous study also informs the reader that the percentages of incomplete colonoscopies were not statistically different among the three age groups, though these proportions were high at slightly $<10 \%$.

One final consideration is that since the current model requires evaluations such as ultrasound and blood assays, it may be more costly and cumbersome as compared to other 
Table 1 Overview of strengths and limitations in study by Jung et al. aimed at identifying high-risk adults younger than 50 years who may be at increased risk of CRC

\begin{tabular}{ll}
\hline Strengths & Large sample of asymptomatic adults $<50$ years $(25 \mathrm{k}+)$ \\
& Excluded adults with family history of CRC \\
& Included two potent CRC risk factors, smoking and obesity \\
& Included biologic-based data points using blood values and hepatic sonography \\
& Extensive screening questionnaire included many factors such as alcohol and exercise \\
Potential & Only group with NNS less than control group of average-risk women were men with all risk factors $45-49$ years; may not \\
limitations & alter current paradigm much \\
& Lack of generalizability due to an ethnically homogeneous population, possible selection bias and obesity defined as 25 or \\
& greater \\
& Addition of laboratory tests makes proposed strategy cumbersome
\end{tabular}

risk models that utilize medical data that can be obtained during a routine office visit. Thus, while the biologic-based factors might add unique information to the other variables that were included, their inclusion does make the strategy less "user friendly." As one expert has stated, prediction models should be a balance between simplicity and accuracy [14].

In summary, the strategy developed by Jung et al. to predict risk of advanced neoplasia in young adults did not identify a group of individuals younger than 45 years who would benefit from screening (Table 1). Nonetheless, the results of this study should motivate investigators to develop models aimed at identifying younger adults at increased risk of CRC. Future study should also include assessments of the benefits of earlier screening with regard to $\mathrm{CRC}$ - versus non-CRC-related morbidity and mortality.

\section{Compliance with ethical standards}

Conflict of interest None.

\section{References}

1. Rex DK, Johnson DA, Anderson JC, et al. American College of Gastroenterology guidelines for colorectal cancer screening 2009 [corrected]. Am J Gastroenterol. 2009;104:739-750.

2. Ahnen DJ, Wade SW, Jones WF, et al. The increasing incidence of young-onset colorectal cancer: a call to action. Mayo Clin Proc. 2014;89:216-224.

3. Jung YS, Yun KE, Chang Y, Ryu S, Park DI. Risk factors such as male sex, smoking, metabolic syndrome, obesity, and fatty liver do not justify screening colonoscopies before age 45. Dig Dis Sci. (Epub ahead of print). doi:10.1007/s10620-015-3912-5.
4. Anderson JC, Messina CR, Dakhllalah F, et al. Body mass index: a marker for significant colorectal neoplasia in a screening population. J Clin Gastroenterol. 2007;41:285-290.

5. Anderson JC, Attam R, Alpern Z, et al. Prevalence of colorectal neoplasia in smokers. Am J Gastroenterol. 2003;98:2777-2783.

6. Bortniker E, Anderson JC. Do recent epidemiologic observations impact who and how we should screen for CRC? Dig Dis Sci. 2015;60:781-794.

7. Imperiale TF, Monahan PO, Stump TE, Glowinski EA, Ransohoff DF. Derivation and validation of a scoring system to stratify risk for advanced colorectal neoplasia in asymptomatic adults: a cross-sectional study. Ann Intern Med. 2015;163:339-346.

8. Jung YS, Ryu S, Chang Y, et al. Risk factors for colorectal neoplasia in persons aged 30 to 39 years and 40 to 49 years. Gastrointest Endosc. 2015;81:e7.

9. Hong SN, Kim JH, Choe WH, et al. Prevalence and risk of colorectal neoplasms in asymptomatic, average-risk screenees 40 to 49 years of age. Gastrointest Endosc. 2010;72:480-489.

10. Imperiale TF, Wagner DR, Lin CY, Larkin GN, Rogge JD, Ransohoff DF. Risk of advanced proximal neoplasms in asymptomatic adults according to the distal colorectal findings. N Engl J Med. 2000;343:169-174.

11. Anderson JC, Latreille M, Messina C, et al. Smokers as a highrisk group: data from a screening population. J Clin Gastroenterol. 2009;43:747-752.

12. Chang LC, Wu MS, Tu CH, Lee YC, Shun CT, Chiu HM. Metabolic syndrome and smoking may justify earlier colorectal cancer screening in men. Gastrointest Endosc. 2014;79:961-969.

13. Chung SJ, Kim YS, Yang SY, et al. Prevalence and risk of colorectal adenoma in asymptomatic Koreans aged 40-49 years undergoing screening colonoscopy. J Gastroenterol Hepatol. 2010;25:519-525.

14. Wells BJ, Kattan MW, Cooper GS, Jackson L, Koroukian S. Colorectal cancer predicted risk online (CRC-PRO) calculator using data from the multi-ethnic cohort study. JABFM. 2014;27:42-55. 